\section{It is time to take timing seriously in clinical genetics}

\author{
György Kosztolányi,1
}

\begin{abstract}
Observations made by molecular techniques on the genome along the individuals' lifetime indicate that the genome in somatic cells displays changes at molecular, cellular, and organismal levels. Timing of genetic events leading to somatic mosaicism and gene expression dynamism results in a highly important variable for comprehending the role of genetics in health and disease. Consideration of time in clinical genetics should be enthusiastically invested into research strategy, interpretation of the results, diagnostic routine, and particularly in ethical discussions.
\end{abstract}

European Journal of Human Genetics (2015) 23, 1435-1437; doi:10.1038/ ejhg.2014.271; published online 24 December 2014
$\mathrm{O}$ ver the first century of Mendelian genetics, up until the recent decades, the central dogma of germline stability has dominated thinking in biology, with continuing refinements in the understanding of inheritance, genetic control of life processes, and genotype-phenotype correlations. Genetics, the science of inheritance, emphasizes the widely accepted view that human beings feature a lifelong genetic stability. The genetic determination inherited from the parents and transmitted to the offspring renders unique identity for every human being. Since the advent of molecular genetics methodology, we have been learning that the basis of this belief is the DNA sequence of the genome, which is transmitted from one generation to the next through the germ line. The base sequence stability of the human genome has been validated by robust molecular genetic techniques, and it serves as an essential supposition not only for the Mendelian rules of inheritance but also for a variety of applied research such as population genetics, evolutionary genetics, ethnological studies, forensic analyses, and so on. Moreover, the stability of the human genome is the fundamental supposition of clinical genetics. Any mutations, that is, significant differences from the supposed 'normal' genomic architecture detected by cytogenetic or molecular technologies, will result in aberrations in the phenotype. The stability of the germline genome is

the base of Mendelian genetics, at least in transgenerational aspect of genetics.

In recent work, we recommended ${ }^{1}$ to extend the approach to genetic disorders with a developmental viewpont, which, in contrast to the transgenerational view, focuses on how the genetic program assembled in the zygote controls the formation of a new individual and how this becomes gradually manifested during the life span. In the developmental aspect of genetics, however, genetic stability of a human being should be seen differently. This review illuminates the issue particularly focusing on time, a neglected variable in our thinking in clinical genetics, which should be considered seriously, if we are to approach the genetic causation of diseases at an individual level with a developmental view.

The role of time and its relation to genetic stability during the lifetime are discussed by reviewing somatic mosaicism and gene expression dynamics.

\section{SOMATIC MOSAICISM}

We usually think of cells taken from a person for genetic analysis as representing the individual's whole organism by sharing the same genome. ${ }^{2}$ However, if we approach genetics in a developmental view, this supposition has to be modified by the knowledge that during the whole life, from embryonic differentiation to death, the genome of every cell in the organism is subject to continuous

${ }^{1}$ Department of Medical Genetics, University of Pécs, Pécs, Hungary

*Correspondence: Professor G Kosztolányi, Department of Medical Genetics, University of Pécs, József A.u. 7 H-7623 Pécs, Hungary. Tel: +36 72535 976; Fax: +36 72535 972; E-mail: kosztolanyi.gyorgy@pte.hu

Received 8 July 2014; revised 14 October 2014; accepted 10 November 2014; published online 24 December 2014 environmental exposure. The fact that mutation of the germline genome may occur and that the cell that is affected may survive has first been demonstrated by chromosomal investigation, leading to the coexistence of two or more cell lines in the organism (somatic mosaicism). ${ }^{3}$ In mosaicism, the proportion of cells carrying the mutation depends on the time the mutation occurred during differentiation and postnatal life, occasionally residing only in certain tissues (tissue mosaicism). The mosaicism may also disappear during development. Essentially, it means that the normal chromosome discovery in the blood does not exclude the possibility of mosaicism.

In addition to chromosomal abnormalities, mosaicism has been documented in a growing number of monogenic disorders caused by somatically acquired mutations. The mitochondrial genome also accumulates alterations throughout the lifetime of an individual. According to his catalog reviewing observations on somatic mosaicism, Erickson ${ }^{4}$ suggests that depending on the disorder and the class of mutation of cases for which there are sufficient numbers of patients studied, $6-20 \%$ are due to somatic mutation. The detection of mosaicism in single-gene disorders depends mainly on the method used for identifying the deleterious mutations. Somatic mutations leading to clonal mosaicism are likely to be the primary triggering event in many, if not all, cancers.

Evidence is mounting that cells of an individual do not share the same genome even in relation to genomic variants such as single-nucleotide polymorphisms and copy number variants. ${ }^{5,6}$ The genomic differences in DNA variants between monozygotic twins resulting from mitotic changes during differentiation and development may lead to discordance in common complex disorders. ${ }^{7}$ Recent investigations revealed also intraindividual genetic differences. In the sequence of the genome between individual sperm cells obtained from one person, Wang et al. ${ }^{8}$ demonstrated significant genetic differences between them, confirming that each sperm is indeed unique. A single tumor can have many different mutations at various locations; this finding highlights the difficulty of treating cancer based solely on one biopsy. ${ }^{9}$

The techniques required to identify all types of genomic variants are far beyond the capacity of usual testing methods. Moreover, the ability of even cell separation techniques to detect mosaicism is poor, particularly when the mosaicism rate is low. Nevertheless, extending molecular techniques into various tissues has proved that mosaicism is much 
more common than we originally thought. Consequently, cells in a person do not share the same genome. The genetic make-up of an individual may vary during lifetime; it is not the same in an adult than it was in the individual's infancy or childhood, one half century earlier in life. One may postulate that our genome in somatic cells is far less stable when compared with germ cells and that the stability of our genome should be viewed accordingly in developmental aspect of diseases. Recently, Shipony et al. ${ }^{10}$ showed that human embryonic stem cells preserve their epigenetic state by balancing antagonistic processes, whereas the somatic epigenome is more vulnerable to noise, as random epimutations can accumulate to massively perturb the epigenomic ground state.

\section{DYNAMISM IN GENE EXPRESSION}

Commitment of multipotent cells toward differentiated cell types and tissues during development, and the continuous reactions of cells to their fluctuating environment with changes in gene expression by epigenetics regulation must be dynamically coordinated by time. ${ }^{11}$ Genomic studies in plant cells have already revealed that gene expression is highly flexible and that light or temperatures induce massive reprogramming of the transcriptome. ${ }^{12}$ The temporal patterns of gene expression in mammalian cells are largely unknown, because data measuring the cells' activity over different points in time from a variety of tissues and under different conditions are difficult to obtain. Most assays provide a single snapshot of cellular regulatory events, whereas a time series capturing how such processes change would be preferable. ${ }^{13}$ Development of RNA-specific dynamic methods for identifying temporal changes in gene expression (RNA-seq) will ideally reveal a snapshot of RNA presence and quantity from a genome at a given point in time. ${ }^{14}$

Yet, some recent microarray studies could already demonstrate that expression timing has a vital role in cellular responses to cope with an environmental perturbation. ${ }^{15}$ The gene for prolactin, for example, was reported to display dynamic expression and pulsating transcription with implications for cellular differentiation. ${ }^{16}$ Jouffe et al. ${ }^{17}$ observed rhythmic diurnal expression of many of the components required for translation initiation, indicating that the circadian clock coordinates the regulation of the biogenesis of ribosomes. The characterization of gene regulation revealed an unexpected nonlinear transcriptional program and indicates that the function of regulatory elements is complex on many time scales ranging from milliseconds to hours. ${ }^{18}$ Disrupting the timing of the sleep-wake cycle can markedly affect the circadian rhythm of gene expression. ${ }^{19}$ In their analysis, Chen et al. ${ }^{20}$ combined various omics profiling of a single individual over a 14-month period and revealed extensive, dynamic changes in diverse molecular components and biological pathways across healthy and diseased conditions.

Although X-chromosome inactivation (XCI), also an epigenetic phenomenon, is presumably executed very early during embryogenesis in somatic cells, recent works detected that skewing in XCI may be established also in early childhood and may become more prevalent with age. ${ }^{21}$ In populations of women past 55-60 years of age, an increased degree of skewing was found. ${ }^{22}$ These data have implications for our understanding about sex differences in complex diseases and the potential causes of phenotypic discordance between monozygotic female twins.

The role of time in (epi) genetic modification is better understood relative to development, which is controlled by temporally organized variable combinations of gene activity. However, timing and environmental epigenetics should be additionally considered in disease causation, first, in common complex disorders in which environmental provocations are a well-known triggering factor for manifestation of the inherited polygenic susceptibility. Advances in molecular techniques will place gene expression analysis into routine diagnostic use, completing base sequence determination, within the next few years. The scientific and clinical benefits of gene expression approach will, however, be accompanied by concerns about how the gene expression profile of an individual determined in a given point of lifetime represents the health likelihood and disease risk. It may prove beneficial to know whether or not the genes currently in research are involved in functional processes directly relevant to the phenotype. In theory, the use of functional gene annotations will represent the utmost support for this specific task. ${ }^{23}$ System-wide approaches are being developed to construct and mine integrated databases of human genes and transcripts for global analysis of biological processes and clinical outcomes. One of the major future challenges for the ENCODE project ${ }^{24}$ whose primary objective is to orchestrate the entire encyclopedia of DNA elements of the human genome ideally capturing the dynamic aspects of gene regulation.

\section{IMPLICATION OF ETHICAL-LEGAL ISSUES}

The lifetime dynamic changes in the genome of humans should be implicated not only in research activity but also in clinical use of genetic testing. All instruments, guidelines, or documents regulating ethical/legal issues in genetics are founded on the premise that the genomic data do not change during lifetime. The increasing knowledge on time-dependent omics changes in composition and expression of the genome during the life of a person poses the question as to whether or not this notion can be sustained. Instead of lifelong stability, plasticity is an important attribution of the human genome, meaning that any genetic test result derived from certain cells taken at a given point of lifetime of a person may not produce identical results derived from other groups of cells taken years (or decades) earlier or thereafter. Indeed, longitudinal personalized omics profiling approach across multiple time points for the same individual indicates that the predictive value of a genetic test result for lifelong health likelihood and disease risk is restrictive and ought to be considered on a strictly individual basis. ${ }^{20}$ Bunnik et al. ${ }^{25}$ suggest a tieredlayered-staged model for informed consent in personal genome testing, spotlighting various limitations of the tests including the fact that the test results may change over time. Screening studies in a cohort of patients with steroid-resistant nephrotic syndrome suggest an inverse relationship of the rate of genetic diagnosis with age, the prevalence figures of genetic causes ranging from $100 \%$ downward to $57 \%, 36 \%$, and $14 \%$ for congenital-onset, infantile-onset, childhoodonset, and adult-onset cases, respectively, suggesting that the clinical utility of genetic testing for a diagnostic purpose depends on the age of the patients. ${ }^{26,27}$

Accordingly, the current guidelines on ethical issues involving the definition of personal data, privacy, identifiability, and confidentiality, moreover, controlling biobanking, may possess reconsideration to meet the concept of dynamic changes in the genome during the lifetime. ${ }^{28,29}$

\section{IN CONCLUSION}

Observations made by molecular techniques on the human genome along time scale indicate that the genome in developmental view of genetics, that is, focusing on how the genetic program assembled in the zygote manifests during the individual's lifetime, is not static and displays changes at the molecular, cellular, and organismal levels. There are two definitive reasons as to why timing in 
clinical genetics should be taken seriously. First, the time when disease-causing de novo mutations occur during development, establishing mosaicism, contributes significantly to the phenotype. Second, the knowledge accumulated suggests that gene expression in living cells changes along time. Timing of genetic events such as mutations leading to somatic mosaicism and gene expression dynamism results in an essential variable for understanding the role of genetics in health and disease, and it will likely affect the personalized genomic medicine in the future. From reductionist approaches in biology, researchers should focus on system-level properties of living organisms, including dynamism, well beyond the circadian clock. ${ }^{30}$ It is worth investigating whether the genome's plasticity in the function of time, the fourth dimension of the genetic control of life, may be a possible reason behind the 'missing heritability'. It is suggested the developmental way of thinking, specifically making the effort and investing the time intensively in genetics, implementing into future research strategy, study protocol, interpretation of the results, diagnostic routine, including genuine esthical discussions, since the pillar of lifelong genetic stability should be reconsidered.

\section{CONFLICT OF INTEREST}

The author declares no conflict of interest.
1 Kosztolanyi G, Cassiman J-J: The medical geneticist as expert in the transgenerational and developmental aspects of diseases. Eur J Hum Genet 2010; 18: 1075-1076.

2 Macosko EZ, McCarroll SA: Exploring the variation within. Nat Genet 2012; 44: 614-616.

3 De S: Somatic mosaicism in healthy human tissues. Trends in Genet 2011; 27: 2017-2223.

4 Erickson RP: Somatic gene mutation and human disease other than cancer: an update. Mut Res 2010; 705: 96-106.

5 Biesecker LG, Spinner NB: A genomic view of mosaicism and human disease. Nat Rev Genet 2013; 14: 307-320.

6 Lupski JR: Genome mosaicism-one human, multiple genomes. Science 2013; 341: 358-359.

7 Maiti S, KHBG Kumar, Castellani CA, O'Reilly R, Singh SM: Ontogenetic de novo copy number variations (CNVs) as a source of genetic individuality: studies on two families with $\mathrm{mz}$ twins for schizophrenia. PLoS One 2011; 6: e17125.

8 Wang J, Fan HC, Behr B, Quake SR: Genome-wide single-cell analysis of recombination activity and de novo mutation rates in human sperm. Cell 2012; 150: 402-412.

9 Gerlinger M, Rowan AJ, Horswell S et al: Intratumor heterogeneity and branched evolution revealed by multiregion sequencing. N Engl J Med 2012; 366: 883-892.

10 Shipony Z, Mukamel Z, Cohen NM et al: Dynamic and static maintenance of epigenetic memory in pluripotent and somatic cells. Nature 2014; 513: 115-119.

11 Chechik G, Koller D: Timing of gene expression responses to environmental changes. J Comput Biol 2009; 16: 279-290.

12 Deal RB, Henikoff S: Gene regulation: a chromatin thermostat. Nature 2010; 463: 887-888.

13 Ecker JR: Serving up a genome feast. Nature 2012; 489: 52-53.

14 Oh S, Song S, Dasgupta N, Grabowski G: The analytical landscape of static and temporal dynamics in transcriptome data. Front Genet 2014; 5: 35.

15 Morgan AA, Dudley JT, Deshpande T, Butte AJ: Dynamism in gene expression across multiple studies. Physiol Genomics 2010; 40: 128-140.

16 Featherstone K, Harper CV, McNamara A et al: Pulsatile patterns of pituitary hormone gene expression change during development. J Cell Sci 2011; 124: 3484-3491.
17 Jouffe C, Cretenet G, Symul L et al: The circadian clock coordinates ribosome biogenesis. PLOS Biol 2013; 11: e1001455.

18 Voss C, Hager GL: Dynamic regulation of transcriptional states by chromatin and transcription factors. Nat Rev Genet 2013; 15: 69-81.

19 Archer SN, Laing EE, Möller-Levet CS et al: Mistimed sleep disrupts circadian regulation of the human transcriptome. Proc Natl Acad Sci USA 2013; 111: E682-E691

20 Chen R, Mias GI, Li-Pook-Than J et al: Personal omics profiling reveals dynamic molecular and medical phenotypes. Cell 2012; 148: 1293-1307.

21 Wong CCY, Caspi A, Williams B, Houts R, Craig IW, Mill J: A longitudinal twin study of skewed $X$ chromosome-inactivation. PLOS One 2011; 6: e17873.

22 Mengel-From J, Thinggaard M, Christiansen L, Vaupel JW, Orstavik KH, Christensen K: Skewed X inactivation and survival: a 13-year follow-up study of elderly twins and singletons. Eur J Hum Genet 2012; 20: 361-364.

23 Piro RM, Ala U, Molineris I et al: An atlas of tissuespecific conserved coexpression for functional annotation and disease gene prediction. Eur J Hum Genet 2011; 19: 1173-1180.

24 ENCODE Project Consortium: An integrated encyclopedia of DNA elements in the human genome. Nature 2012; 489: 57-74

25 Bunnik EM, Janssens AC, Schermer MH: A tieredlayered-staged model for informed consent in personal genome testing. Eur J Hum Genet 2013; 21: 596-601.

26 Lipska BS, Ranchin B, latropoulos P et al: Genotypephenotype associations in WT1 glomerulopathy. Kidney Int 2014; 85: 1169-1178.

27 Santín S, Bullich G, Tazón-Vega B et al: Clinical utility of genetic testing in children and adults with steroidresistant nephrotic syndrome. Clin J Am Soc Nephrol 2011; 6: 1139-1148.

28 Knoppers BM, Dove ES, Litton J-E, Nietfeld JJ: Questioning the limits of genomic privacy. Am J Hum Genet 2012; 91: 577-579.

29 van El CG, Cornel MC, Borry P et al: Whole-genome sequencing in health care. Recommendations of the European Society of Human Genetics. Eur J Hum Genet 2013; 21: 580-584.

30 Hogenesch JB, Ueda HR: Understanding systems-leve properties: timely stories from the study of clocks. Nat Rev Genet 2011; 12: 407-416. 